\title{
Sequential Memory: A Putative Neural and Synaptic Dynamical Mechanism
}

\author{
Gustavo Deco' ${ }^{1}$ and Edmund T. Rolls ${ }^{2}$
}

\begin{abstract}
A key issue in the neurophysiology of cognition is the problem of sequential learning. Sequential learning refers to the ability to encode and represent the temporal order of discrete elements occurring in a sequence. We show that the short-term memory for a sequence of items can be implemented in an autoassociation neural network. Each item is one of the attractor states of the network. The autoassociation network is implemented at the level of integrate-and-fire neurons so that the contributions of different biophysical mechanisms to sequence learning can be investigated. It is shown that if it is a property of the synapses or neurons that support each attractor state that they adapt, then everytime the network is made quiescent (e.g., by inhibition), then the
\end{abstract}

\section{INTRODUCTION}

A key issue in the neurophysiology of cognition is the problem of sequential learning. Sequential learning refers to the ability to encode and represent the temporal order of discrete elements occurring in a sequence. Many of the behaviors that we produce are sequential in nature. The learning of serial order is fundamental to all forms of skilled behavior from motor sequences (e.g., playing the piano, dancing, drawing) to language (Averbeck, Chafee, Crowe, \& Goergopoulos, 2002; Conway \& Christiansen, 2001; Dell, Burger, \& Svec, 1997). Complex sequential learning is very evident in language acquisition and production.

A major contribution to the complex problem of serial order learning was provided by Lashley (1951). Lashley's theory suggests a model of sequential learning that is based on parallel response activation. This means that the temporal serial order in behavior assumes a cotemporal neural representation of all serial elements before action begins. Averbeck et al. (2002) supported this theory by training two monkeys to copy simple geometrical shapes, which they drew as a series of movement segments, and investigated the neural representation of the serial order of these segments in the prefrontal

${ }^{1}$ ICREA and Universitat Pompeu Fabra, ${ }^{2}$ University of Oxford attractor state that emerges next is the next item in the sequence. We show with numerical simulations implementations of the mechanisms using (1) a sodium inactivation-based spike-frequency-adaptation mechanism, (2) a $\mathrm{Ca}^{2+}$-activated $\mathrm{K}^{+}$current, and (3) short-term synaptic depression, with sequences of up to three items. The network does not need repeated training on a particular sequence and will repeat the items in the order that they were last presented. The time between the items in a sequence is not fixed, allowing the items to be read out as required over a period of up to many seconds. The network thus uses adaptation rather than associative synaptic modification to recall the order of the items in a recently presented sequence.

cortex, an area with an established role in serial ordering of motor actions and working memory (Fuster, 2000; Goldman-Rakic, 1987). In fact, lesions of the prefrontal cortex impair the sequential learning of visual stimuli in monkeys (Petrides, 1991), and disrupt serial temporal motor integration in humans (Luria, 1966). Averbeck et al. provided evidence that all serial elements of a movement sequence are represented in an orderly fashion in the prefrontal cortex before the action begins. In addition, Dell et al. (1997) studied a connectionist abstract neural network model of language production based on Lashley's arguments, which was able to account for serial-order errors in speech. The most basic characteristic of their model was its capacity to activate the present, deactivate the past, and prepare for the future.

The aim of the present work is to introduce a detailed and plausible model of serial order memory at the neurobiological level. A crucial contribution of the present model, and a difference from Lashley's arguments, is that we assume that the temporal coding of actions before their execution can be understood at the level of internal and intrinsic biophysical variables of neurons related to spike-frequency mechanisms. These neuronal processes are able to activate the present, deactivate the past, and prepare for the future.

We propose three putative and potentially alternative neuron-level mechanisms to implement the required processing, namely, (1) a sodium inactivation-based spike- 
frequency-adaptation mechanism, (2) a $\mathrm{Ca}^{2+}$-activated $\mathrm{K}^{+}$current, and (3) short-term synaptic depression. We investigate here a mechanism that, by utilizing an attractor autoassociative network with recurrent connections (Rolls \& Deco, 2002; Rolls \& Treves, 1998; Hopfield, 1982), can maintain the sequence item that is current in a continuing active state of firing until the next item stored in the same attractor is turned on. To implement this model in a biologically plausible form, the processes occurring at the alpha-amino-3-hydroxy-5-methyl-4isoxazolepropionic acid (AMPA), $N$-methyl-D-aspartate (NMDA), and gamma-aminobutyric acid (GABA) synapses are dynamically modeled in an integrate-and-fire implementation to produce realistic spiking dynamics (Deco \& Rolls, 2003; Brunel \& Wang, 2001). This also enables the synaptic adaptation that is one of the putative mechanism to be realistically implemented. The NMDA receptors provide no particular role in the mechanism for sequence memory described here, but are included in the simulations for biological accuracy, and because of their long time constant and nonlinearity, they do make a useful contribution to the dynamical stability of integrate-and-fire networks.

\section{RESULTS}

\section{Model}

\section{Prefrontal Architecture}

We explicitly model the level of processes occurring at the AMPA, NMDA, and GABA synapses in the integrateand-fire implementation to produce realistic spiking dynamics. We follow the neurodynamical framework introduced by the authors (Corchs \& Deco, 2002; Deco \& Lee, 2002; Rolls \& Deco, 2002; Deco \& Zihl, 2001) and the integrate-and-fire neuronal framework introduced and studied by Brunel and Wang (2001). We incorporate shunting inhibition (Battaglia \& Treves, 1998; Rolls \& Treves, 1998) and inhibitory-to-inhibitory cell synaptic connections (Brunel \& Wang, 2001), which are useful in maintaining stability of the dynamical system, and incorporate appropriate currents to achieve low firing rates (Brunel \& Wang, 2001; Amit \& Brunel, 1997). We assume in the single attractor network architecture investigated the existence of different neuronal populations or pools, each of which corresponds to an item or element of the sequence (a visual stimulus, a simple motor action, a phoneme, or a syntactic element). By a single attractor network, we mean a single autoassociation network with firing that can reflect at any one time one of a number of different attractor memory states stored in the network by synaptic modification (Rolls \& Deco, 2002; Rolls \& Treves, 1998). Each memory, item, or attractor state involves the firing of one of the populations (also called pools) of neurons in the network. We show that a single network is sufficient in terms of architecture for operation of the model. Because the prefrontal cortex is implicated in some types of sequence memory, we use the term prefrontal architecture above, but a network of the type we describe could be implemented in a number of different brain areas. In this section, we describe the architecture and operation of the model and provide a full mathematical specification of the model, and the neuronal parameters used, in the Appendix section.

A conceptual overview of the single autoassociation network architecture is shown in Figure 1. The different specific pools are activated sequentially because there is partial adaptation in a neuronal pool when it is active, so that after a global inhibition signal shuts down the current attractor, the least recently activated neuronal
Figure 1. Architecture of the model. There is a single attractor network with the items T1-Tn that can be part of a sequence stored using AMPA and NMDA receptors in the recurrent collateral connections. There is a pool of inhibitory interneurons using GABA as the transmitter. In addition, there is a non-specific pool of neurons with random spiking activity.

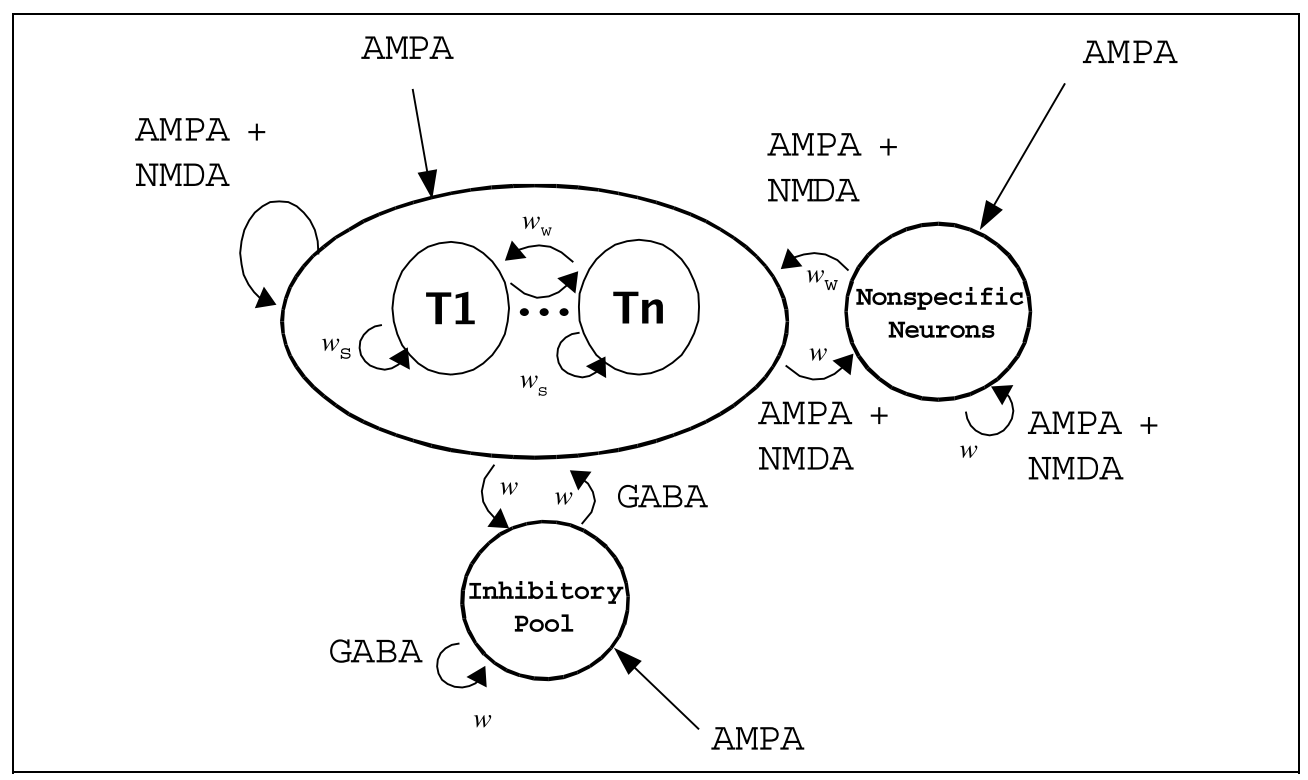


pool emerges into activity. The adaptation mechanism may be at the neuronal or synaptic level. If the network is presented with a short sequence of stimuli to remember, it will retrieve them in the order in which they were just presented.

In the case of a sodium inactivation-based spikefrequency-adaptation mechanism, the internal probability of spike emission near the threshold will be the relevant variable that correlates with the temporal order in the sequence memorized. In the case of $\mathrm{Ca}^{2+}$-activated $\mathrm{K}^{+}$current, the $\mathrm{Ca}^{2+}$ concentration inside the membrane of the neurons is the relevant variable that correlates with the temporal order in the sequence memorized. Last, in the case of short-term synaptic depression, the probability of neurotransmitter release is the relevant variable that correlates with the temporal order in the sequence memorized.

The network contains $N_{\mathrm{E}}$ (excitatory) pyramidal cells and $N_{\mathrm{I}}$ inhibitory interneurons. In our simulations, we use $N_{\mathrm{E}}=800$ and $N_{\mathrm{I}}=200$, consistent with the neurophysiologically observed proportion of $80 \%$ pyramidal cells versus 20\% interneurons (Rolls \& Deco, 2002; Abeles, 1991). In the network, the neurons are fully connected (with synaptic strengths as specified below). Neurons in the cortical network shown in Figure 1 are clustered into populations or pools. Each pool of excitatory cells contains $f N_{\mathrm{E}}$ neurons, where $f$ is the fraction of the excitatory neurons in any one population (in our simulations, $f=0.1$ ). There are two different types of pool: excitatory and inhibitory.

There are two subtypes of excitatory pool, namely, specific and nonselective. Specific pools encode, for example, the identity of a visual object, or a phoneme, or a simple motor action to be remembered as a part of a temporal sequence. The remaining excitatory neurons are in a nonselective pool. [These neurons have some spontaneous firing and help to introduce some noise into the simulation, which aids in generating the almost Poisson spike firing patterns of neurons in the simulation that are a property of many neurons recorded in the brain (Compte et al., 2003; Brunel \& Wang, 2001)]. All the inhibitory neurons are clustered into a common inhibitory pool, so that there is global competition throughout the network. We assume that the synaptic coupling strengths between any two neurons in the network act as if they were established by Hebbian learning, that is, the coupling will be strong if the pair of neurons have correlated activity and weak if they are activated in an uncorrelated way. As a consequence, neurons within a specific excitatory pool are mutually coupled with a strong weight $\left(w_{\mathrm{s}}=2.1\right)$. Neurons in the inhibitory pool are mutually connected with an intermediate weight $(w=1$, forming the inhibitory to inhibitory connections that are useful in achieving nonoscillatory firing). They are also connected with all excitatory neurons with the same intermediate weight $(w=1)$. The connection strength between two neurons in two different specific excitatory pools is weak and given by $w_{\mathrm{w}}=1-f\left(w_{\mathrm{s}}-1\right) /(1-f)$. Neurons in a specific excitatory pool are connected to neurons in the nonselective pool with a feed-forward synaptic weight $(w=1)$ and a feedback synaptic connection of weight $\left(w_{\mathrm{w}}\right)$. Each neuron (pyramidal cells and interneurons) receives $N_{\text {ext }}=800$ excitatory AMPA synaptic connections from outside the network. The external inputs are given by a Poisson train of spikes. To model the background, spontaneous activity of neurons in the network (Brunel \& Wang, 2001), we assume that Poisson spikes arrive at each external synapse with a rate of $3 \mathrm{~Hz}$, consistent with the spontaneous activity observed in the cerebral cortex (Rolls \& Treves, 1998; Wilson, O'Scalaidhe, \& Goldman-Rakic, 1994). In other words, the effective external spontaneous background input rate of spikes to each cell is $v_{\text {ext }}=N_{\text {ext }} \times 3 \mathrm{~Hz}=2.4 \mathrm{kHz}$.

We use leaky integrate-and-fire neurons for modeling the excitatory pyramidal cells and the inhibitory interneurons. The synaptic inputs to an integrate-and-fire neuron are basically described by a capacitor $\left(C_{\mathrm{m}}\right)$ connected in parallel with a resistor $\left(R_{\mathrm{m}}\right)$ through which currents are injected into the neuron. These current injections produce excitatory or inhibitory postsynaptic potentials (EPSPs and IPSPs, respectively). These potentials are integrated by the cell, and if a threshold $\theta$ is reached, a $\delta$ pulse (spike) is fired and transmitted to other neurons, and the potential of the neuron is reset. The incoming presynaptic $\delta$ pulse current from another neuron is low-pass filtered by the synaptic and membrane time constants, and an EPSP or IPSP is generated in the one-compartment neuronal model.

The synaptic current flows into the cells are mediated by three different families of receptors. The recurrent excitatory postsynaptic EPSPs are mediated by AMPA and NMDA receptors. These two glutamatergic excitatory synapses are on the pyramidal cells and on the interneurons. The external inputs (background, sensory input, or external top-down interaction from other areas) are mediated by AMPA synapses on pyramidal cells and interneurons. Inhibitory GABAergic synapses on pyramidal cells and interneurons yield the corresponding IPSPs. The mathematical descriptions of each synaptic channel and the corresponding parameters are provided in the Appendix section.

All neuronal and synaptic equations were integrated using the second-order Runge-Kutta method, with an integration step of $\mathrm{d} t=0.1 \mathrm{msec}$. Checks were performed to show that this was sufficiently small. For the neural membrane potential equations, interpolation of the spike times and their use in the synaptic currents and potentials were taken into account following the prescription of Hansel, Mato, Meunier, and Neltner (1998) to avoid numerical problems due to the discontinuity of the membrane potential and its derivative at the spike firing time. The external trains of Poisson spikes were generated randomly and independently. 

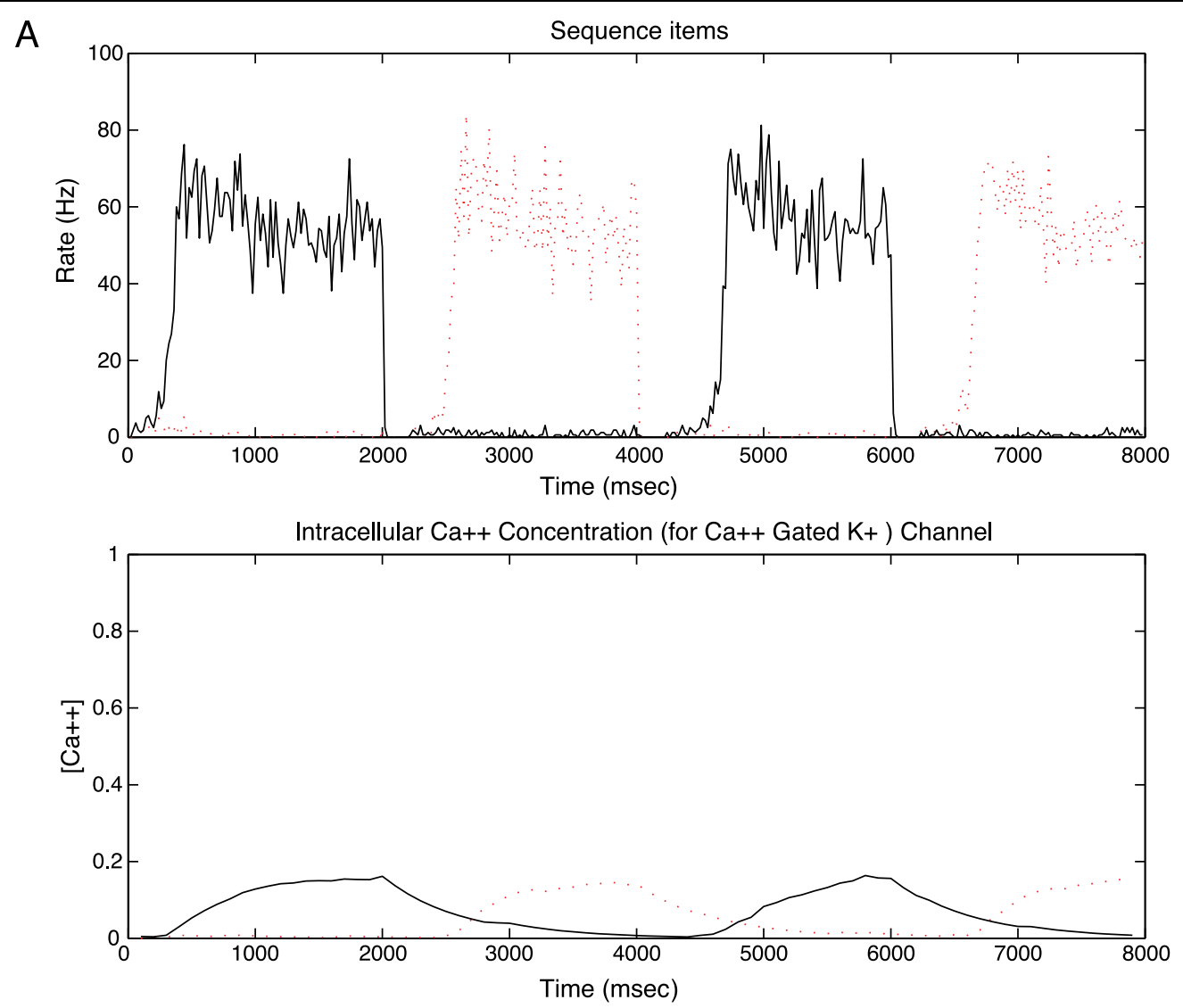

B

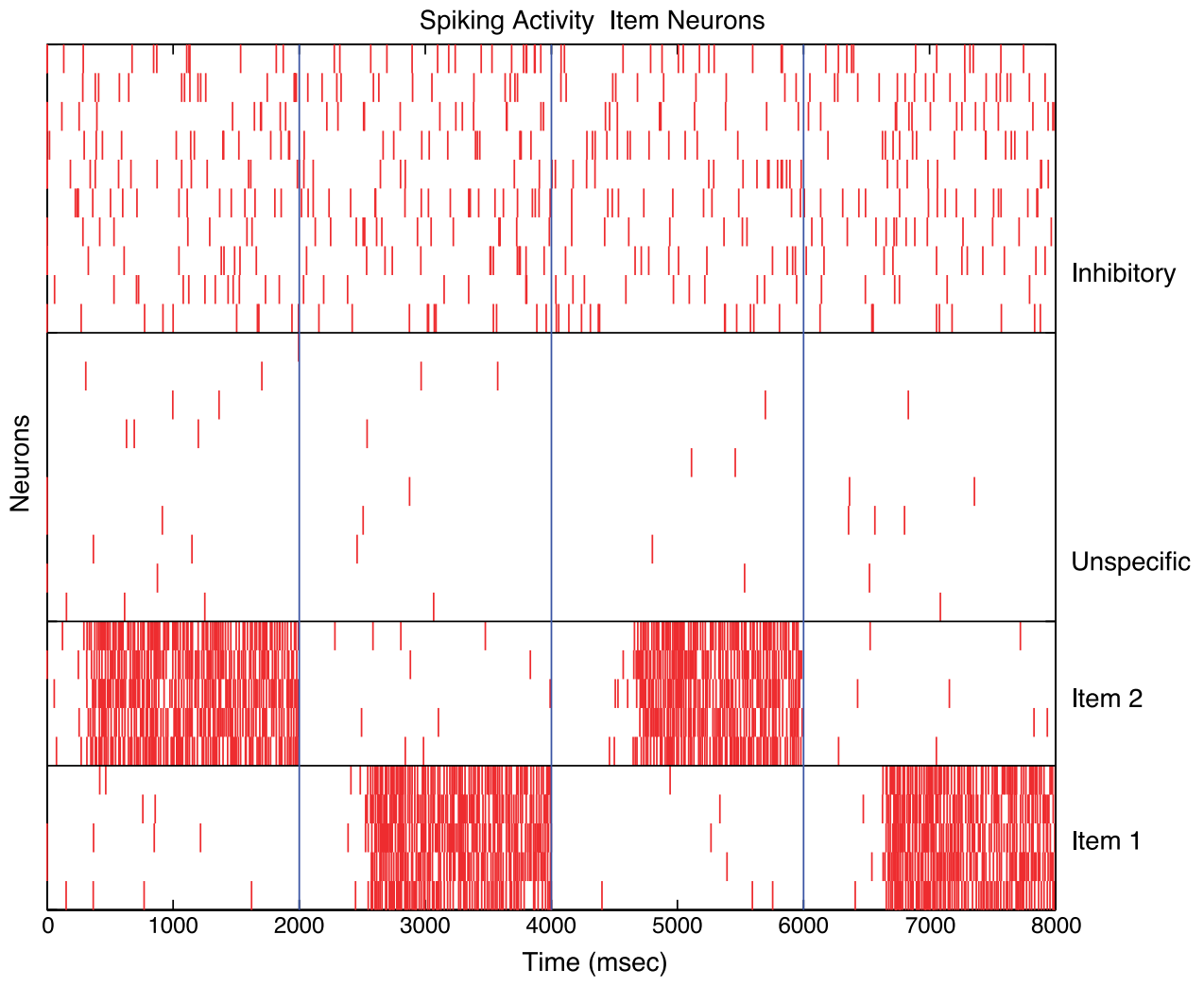




\section{Operation of Sequence Learning}

To achieve the sequential activation of specific pools in the network, we assume that the attractor state is reset periodically via a nonspecific global inhibitory signal. In our implementation, we implemented the effects of this inhibitory signal by increasing for $200 \mathrm{msec}$ the external AMPA input to the inhibitory pool of the network. (The increase was from $v_{\text {ext }}$ to $v_{\text {ext }}+\lambda_{\text {Reset }}$ with $\lambda_{\text {Reset }}=$ $900 \mathrm{~Hz}$, which corresponds to an increase of $1.125 \mathrm{~Hz}$ to each of the 800 external synapses impinging on the neurons of the inhibitory pool. This compares to the mean value of the spontaneous external input of $3 \mathrm{~Hz}$ per synapse.) This increased the global inhibition, and suppressed the activity of all the excitatory neuronal pools in the network. We incorporate into the neurons or synaptic connections between the neurons different mechanisms to implement spike-frequency adaptation (with details provided below). This provides a mechanism that implements a temporal memory of the previously activated pool. When the attractor state of the network is shut down by the inhibitory input, then the attractor state that subsequently emerges when firing starts again will be different from the state that has just been present, because of adaptation of the synaptic or neuronal processes that supported the previous attractor state. To assure that one of the specific pools is active, to promote high competition between the possible specific pools (the items in the sequence), we continuously excite externally all specific pools with the same nonspecific input by increasing the external input Poisson firing rate impinging on the excitatory pools of the network (from $v_{\text {ext }}$ to $v_{\text {ext }}+B$ with $B=200 \mathrm{~Hz}$ ). The function of $B$ is thus to maintain as selected the set of items in the sequence being remembered.

We describe now the specific implementation of the spike-frequency-adaptation mechanism that we used in the network.

Sodium inactivation. One implementation used for the simulations was a sodium inactivation-based spikefrequency-adaptation mechanism. A full statistical analysis of a model of sodium inactivation in the framework of integrate-and-fire models was introduced by Giugliano, La Camera, Rauch, Luescher and Fusi (2002) as a realistic candidate for long-lasting nonmonotonic effects in current-to-rate response functions observed in vitro (Rauch, La Camera, Luescher, Seen, \& Fusi, 2003) and associated with spike-frequency mechanisms. The model was called an integrate-and-may-fire model and takes into account the inactivation of sodium channels after spike generation. The integrate-and-fire model is modified by changing the condition that when the membrane potential reaches the threshold $\theta$, the emission of a spike at that time is an event occurring with an activitydependent probability $q$. After the spike emission, the membrane potential is clamped to the value $V_{\text {reset }}$ of $-55 \mathrm{mV}$ for an absolute refractory time, after which the current integration starts again. However, each time the excitability threshold $\theta$ is crossed and no spike has been generated (i.e., an event with probability $1-q$ ), the membrane potential is reset to $H_{2}\left(V_{\text {reset }}<H_{2}<\right.$ threshold $\theta<0$ ) and no refractoriness occurs. Additionally, $q$ is a decreasing function of a slow voltage-dependent variable $\omega(0<\omega<1)$, reminiscent of the sigmoidal voltage dependence of the fast inactivation state variables that characterize conductance-based model neurons:

$$
q=\left[1+e^{\frac{\omega-\omega_{0}}{\sigma_{\omega}}}\right]^{-1}
$$

where $\omega$ evolves by

$$
\tau_{\omega} \frac{d \omega(t)}{d t}=\frac{V(t)}{\theta}-\omega
$$

which corresponds to a first approximation to the average transmembrane electric field experienced by individual ion channels and affecting their population-level activation and inactivation. In our simulations, we used $\tau_{\omega}=9000 \mathrm{msec}, \sigma_{\omega}=0.01, \omega_{0}=0.8563$, and $H_{2}=-52 \mathrm{mV}$. $\mathrm{Ca}^{2+}$-activated $\mathrm{K}^{+}$byperpolarizing currents. We also tried other spike-frequency adapting mechanisms, including $\mathrm{Ca}^{2+}$-activated $\mathrm{K}^{+}$hyperpolarizing currents (Liu \& Wang, 2001). They assume that the intrinsic gating of $\mathrm{K}^{+}$after-hyperpolarizing current $\left(I_{\mathrm{AHP}}\right)$ is fast, and therefore its slow activation is due to the kinetics of the cytoplasmic $\mathrm{Ca}^{2+}$ concentration. This can be introduced in the model by adding an extra current term in the integrate-and-fire model, that is, by adding $I_{\mathrm{AHP}}$ on the right hand of Equation 6 in the Appendix, as follows:

$$
I_{\mathrm{AHP}}=-g_{\mathrm{AHP}}\left[\mathrm{Ca}^{2+}\right]\left(V(t)-V_{K}\right)
$$

where $V_{\mathrm{K}}$ is the reversal potential of the potassium channel. Further, each action potential generates a small amount $(\alpha)$ of calcium influx, so that $I_{\mathrm{AHP}}$ is incremented accordingly. Between spikes, the $\left[\mathrm{Ca}^{2+}\right]$ dynamics is

\footnotetext{
Figure 2. $\mathrm{Ca}^{2+}$-activated $\mathrm{K}^{+}$hyper-polarizing current mechanism. (A) Rate activity and calcium intracellular concentration as a function of time for a temporal sequence of two items. The two items were presented in the order item 2 - item 1 just at the start of the simulation period shown, and retrieval is demonstrated starting at $4000 \mathrm{msec}$. The firing rate and the intracellular calcium concentration for the two populations of neurons are shown. An external signal indicating that the network should move to the next item in the sequence is applied at times 4000 and 6000 msec. This signal quenches the attractor rapidly, and the next attractor takes some time to develop because of the competitive processes taking place between the neurons in the two populations. The rate measure in this and subsequent Figures is the mean firing rate of the neurons in the particular population shown. (B) Rastergrams showing the spiking activity in the neurons in the different populations.
} 
modeled as a leaky integrator with a decay constant $\left(\tau_{\mathrm{Ca}}\right)$. Hence, the calcium dynamics can be described by following system of equations:

$$
\frac{d\left[\mathrm{Ca}^{2+}\right]}{d t}=-\frac{\left[\mathrm{Ca}^{2+}\right]}{\tau_{\mathrm{Ca}}}
$$

If $V(t)=\theta$, then $\left[\mathrm{Ca}^{2+}\right]=\left[\mathrm{Ca}^{2+}\right]+\alpha$ and $V=V_{\text {reset }}$, and these are coupled to the above-mentioned modified equations of the Appendix. The $\left[\mathrm{Ca}^{2+}\right]$ is initially set to be $0 \mu \mathrm{M}, \tau_{\mathrm{Ca}}=600 \mathrm{msec}, \alpha=0.005, V_{\mathrm{K}}=-80 \mathrm{mV}$, and $g_{\mathrm{AHP}}=7.5 \mathrm{nS}$.

Short-term synaptic depression. The synaptic depression mechanism was used following Dayan and Abbott (2002, p. 185). In particular, the probability of transmitter release $P_{\text {rel }}$ was decreased after each presynaptic spike by a factor $P_{\text {rel }}=P_{\text {rel }} \cdot f_{\mathrm{D}}$, with $f_{\mathrm{D}}=0.982$. Between presynaptic action potentials, the release probability $P_{\text {rel }}$ is updated by

$$
\tau_{P} \frac{d P_{\text {rel }}}{d t}=P_{0}-P_{\text {rel }}
$$

with $P_{0}=1$ and $\tau_{\mathrm{P}}=600$ msec.

\section{Simulation of the Model}

To show how the model is able to implement the learning of temporal sequences of items (visual objects, phonemes, simple motor actions, etc.) and specially how Lashley's ideas of cotemporal activation are reflected in the cotemporal activation of intrinsic synaptic (probability of release) or neural (intracellular calcium or probability of spiking due to sodium inactivation) processes, we performed simulations of how the temporal order of a two- or three-element sequence is maintained by using the three different mechanisms mentioned above.

Figure 2 shows simulations corresponding to the use of calcium-dependent AHP potassium currents. Two items formed a sequence. Figure $2 \mathrm{~A}$ plots the rate of average activity in the two specific pools corresponding to the items in the sequence. The network needs an external signal to indicate when it should move to the next item in the sequence. After each switch initiated by the external signal, the correct next item in the sequence is activated. The temporal order is encoded in the intracellular calcium concentration shown in the bottom of Figure 2A. Because the most recently activated pool has a larger amount of intracellular calcium, the inhibitory AHP current causes this pool not to be activated after the switch. Figure $2 \mathrm{~B}$ plots the rastergrams of randomly selected neurons for each pool in the network ( 5 for each specific pool, 10 for the nonselective excitatory pool, and 10 for the inhibitory pool).

Figure 3 shows simulations corresponding to the use of sodium inactivation mechanisms. Three items formed a sequence. Figure 3A plots the rate of average activity in the three specific pools corresponding to the items in the sequence. After each switch, the correct item is activated. The temporal order is encoded in the probability of spiking due to sodium inactivation as shown at the bottom of Figure 3A. Because the most recently activated pool has a lower probability of spiking due to the sodium inactivation, this pool is with high probability not activated after the switch. Figure $3 \mathrm{~B}$ plots the rastergrams of randomly selected neurons for each pool in the network.

Figure 4 also shows simulations corresponding to the use of sodium inactivation mechanisms, but was performed to show that the same architecture after training on several items can remember the sequence of the items even if there are different numbers of items in different sequences. The simulation thus shows that neither the order nor the number of items in the sequence is fixed by the training of the synaptic weights. In this simulation, the number of items is decided (and also which items are in the sequence) by the external bias $\mathrm{B}$ acting only on the pools that represent the items in the sequence. This determines the items that are included in the sequence to be remembered. The order of the items is determined by the order in which the items in the sequence are presented to the network, as a result of the adaptation mechanism described in this article. Figure 4A plots the rate of average activity in the two specific pools corresponding to the items in the sequence. After each switch, the correct item is activated. The temporal order is encoded in the probability of spiking due to sodium inactivation as shown at the bottom of Figure 4A. Because the most recently activated pool has a lower probability of spiking due to the sodium inactivation, this pool is with high probability not activated after the switch. Figure $4 \mathrm{~B}$ plots the rastergrams of randomly selected neurons for each pool in the network. We emphasize that the network and the items stored in the recurrent associative synaptic connections in the network are identical

\footnotetext{
Figure 3. Sodium inactivation mechanism. (A) Rate activity and spiking probability due to sodium inactivation as a function of the time for a temporal sequence of three items. The three items were presented in the order item 3 -item 2 -item 1 just at the start of the simulation period shown, and retrieval is demonstrated starting at time $=15 \mathrm{sec}$. Activity in the three specific corresponding pools is shown. The lower plot shows the probability of spiking which alters due to a sodium inactivation spike frequency adaptation mechanism (see text). An external signal indicating that the network should move to the next item in the sequence is applied at time $=15,20$ and $25 \mathrm{sec}$. (B) Rastergrams showing the spiking activity in the neurons in the different populations.
} 


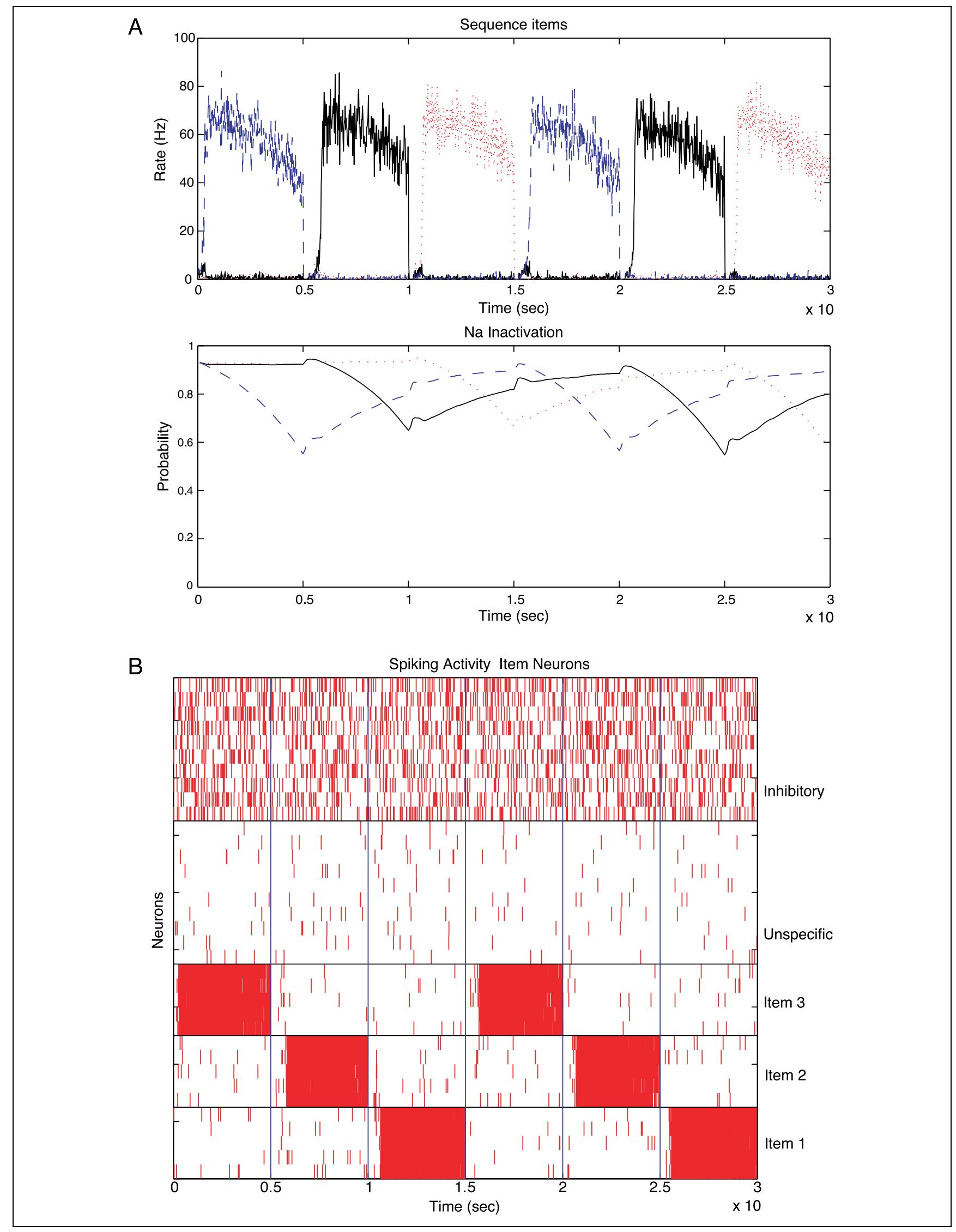


for the simulations shown in Figures 3 and 4. The difference is just that for the simulation in Figure 3, three items were presented as the sequence to be remembered, and the bias input, $B$, was applied to those three items. For the simulation shown in Figure 4, two items were presented as the sequence to be remembered, and the bias input, $B$, was applied to those two items. The bias input, $B$, could originate from a short-term memory attractor or attractors that would hold active the items in the sequence, whereas the network with adaptation described in this article would determine the order in which the items are recalled. The whole of this process could take place for items previously stored in the network everytime a new sequence is needed without any synaptic modification. Indeed, it is a prediction of the model described here that once the items have been trained individually, then the sequence memory could be used to receive and replay different sequences of the items without limit without any further synaptic modification.

Figure 5 shows simulations corresponding to the use of short-term synaptic depression. Two items formed a sequence. Figure $5 \mathrm{~A}$ plots the rate of average activity in the two specific pools corresponding to the items in the sequence. As before, an external signal is used to indicate that the network should move to the next item in the sequence. After each switch, the correct next item is activated. The temporal order is encoded in the probability of transmitter release as shown in the bottom of Figure 5A. Because the most recently activated pool has a lower probability of release, this pool is with high probability not activated after the switch. Figure $5 \mathrm{~B}$ plots the rastergrams of randomly selected neurons for each pool in the network.

\section{DISCUSSION}

The single attractor network implementation of a sequence memory that we describe can store several items in a sequence without repeated training on any one sequence. The memory for the order in which the items were presented is not implemented by long-term associative synaptic modification such as long-term potentiation, but instead by short-term nonassociative adaptation. The adaptation could be of the active neurons, or of the active synapses, as demonstrated. The adaptation must be partial, if the sequence is not to move on automatically, but is to be moved onto the next item in the sequence by an external signal, which quenches the current attractor. If the adaptation is more complete, then the item currently in the attractor will automatically become unstable, and the next least recently active attractor will then become active, without the need for an external signal-producing inhibition to move the network onto the next item in a sequence. In automatic mode, the speed with which the system would move onto the next item need not be very fast, and is a function of how rapidly the adaptation occurs. The fact that the replay of the sequence can be slow is an advantage over some other ways that sequence memory might be implemented that are discussed below. Although the sequence of the items is not stored by associative long-term synaptic modification, each of the items must be trained into the attractor network by long-term associative synaptic modification. The items need not be learned before the sequence is first presented, because the associative synaptic modification could occur rapidly while each item in the sequence is being presented for the first time. The network can replay the sequence of items just presented, but it does not store the sequence itself as a fixed sequence in longterm memory. If the network operates using an external trigger to indicate when the next item should be recalled, this could be provided by something as simple as an excitatory nonspecific input to a region. This will cause extra activation of the inhibitory interneurons and essentially, by competitive interactions implemented through the inhibitory neurons, quench the attractor holding active the current item in the sequence. The magnitude of this reset signal need not be large. For example, the reset can be produced by increasing the external background spontaneous excitatory input to all the 800 external synapses on the neurons in the network from an average of 3 to $3.375 \mathrm{~Hz}$ for $500 \mathrm{msec}$. If the reset signal was applied to a smaller fraction of the neurons, a correspondingly larger change of firing rate would be needed. If we were to keep providing reset inputs, the network would keep on recalling the sequence, providing a mechanism for rehearsal.

In the network we describe, the adaptation must be sufficiently long so that it spans the duration of the whole sequence to be remembered. If the adaptation lasted for only the duration of a single item, then after the second item in a three-item sequence, there would be no preferential recall of the third rather than the first item in the sequence. (In the case of the synaptic adaptation mechanism described in Figure 5, this factor might limit the sequence memory to 3 or 4 items each spaced 2 sec apart in the case of the parameters investigated, but, of course, the value of the relevant param-

Figure 4. Sodium inactivation mechanism. (A) Rate activity and spiking probability due to sodium inactivation as a function of the time for a temporal sequence of two items. The two items were presented in the order item 2 - item 1 just at the start of the simulation period shown, and retrieval is demonstrated starting at $10 \mathrm{sec}$. The two specific corresponding pools are shown. The simulation was identical to that shown in Figure 3 , except that two items instead of three were used. An external signal indicating that the network should move to the next item in the sequence is applied at 10 and $15 \mathrm{~s}$. (B) Rastergrams showing the spiking activity in the neurons in the different populations. 

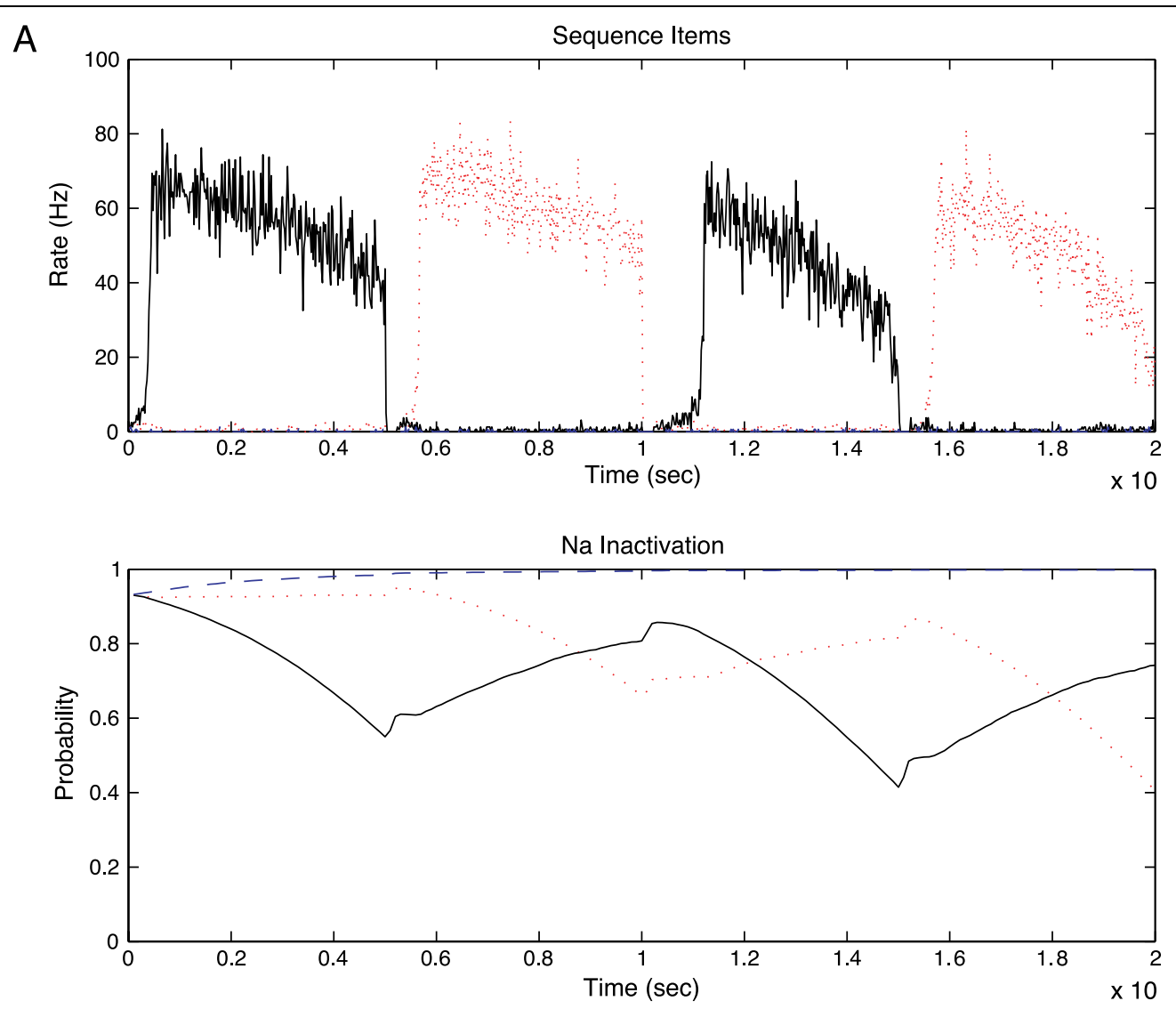

B

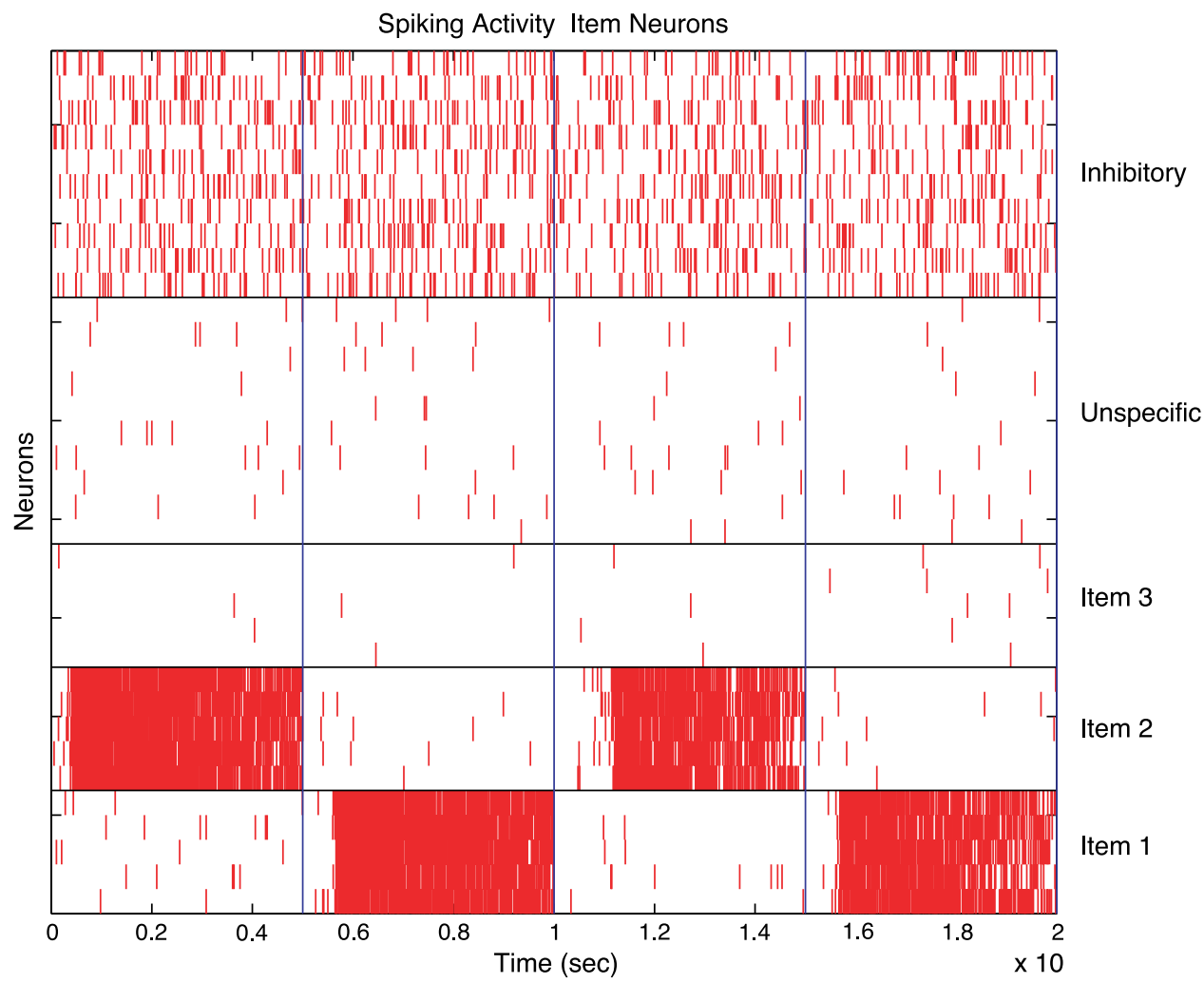



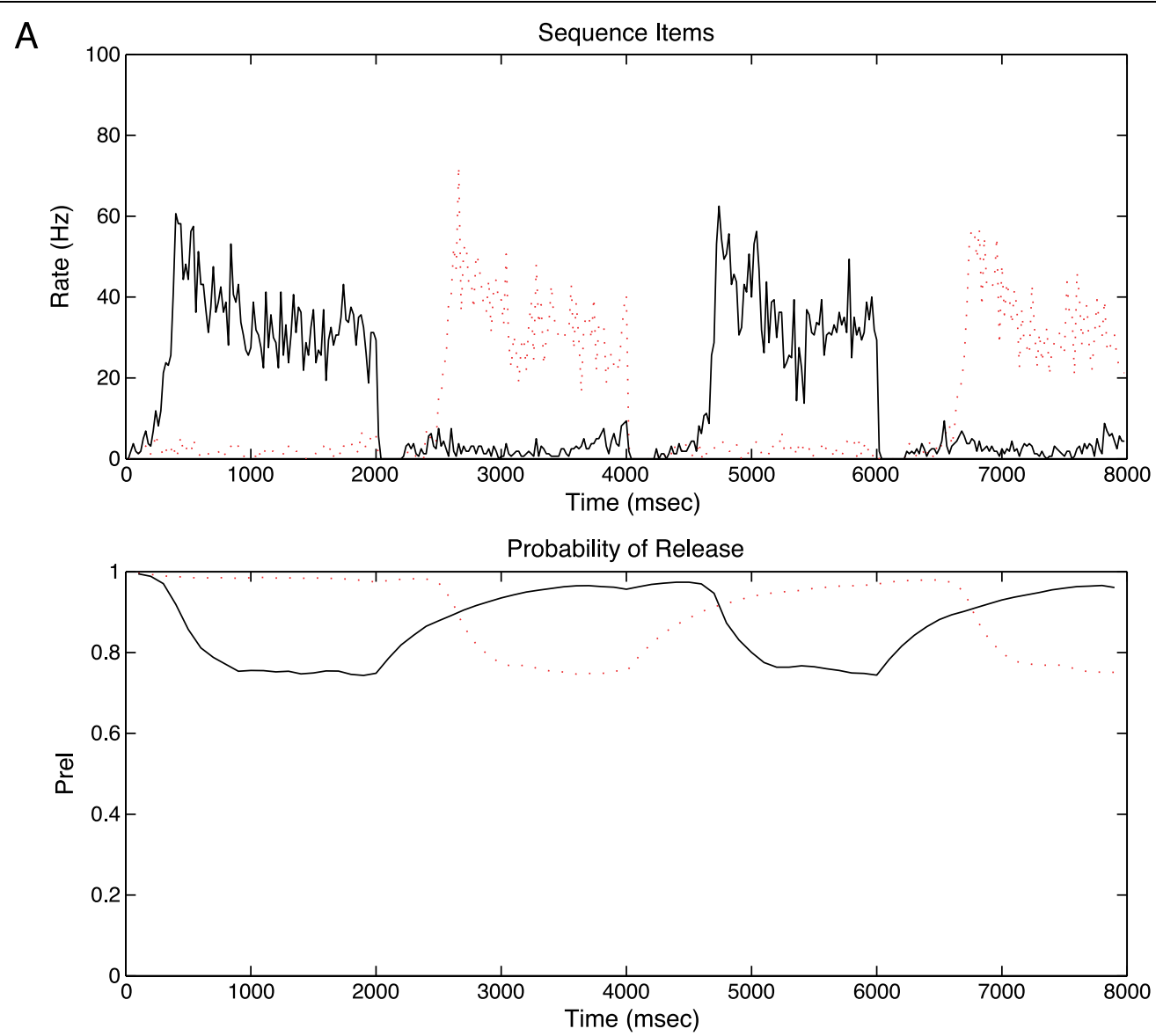

B Spiking Activity Item Neurons

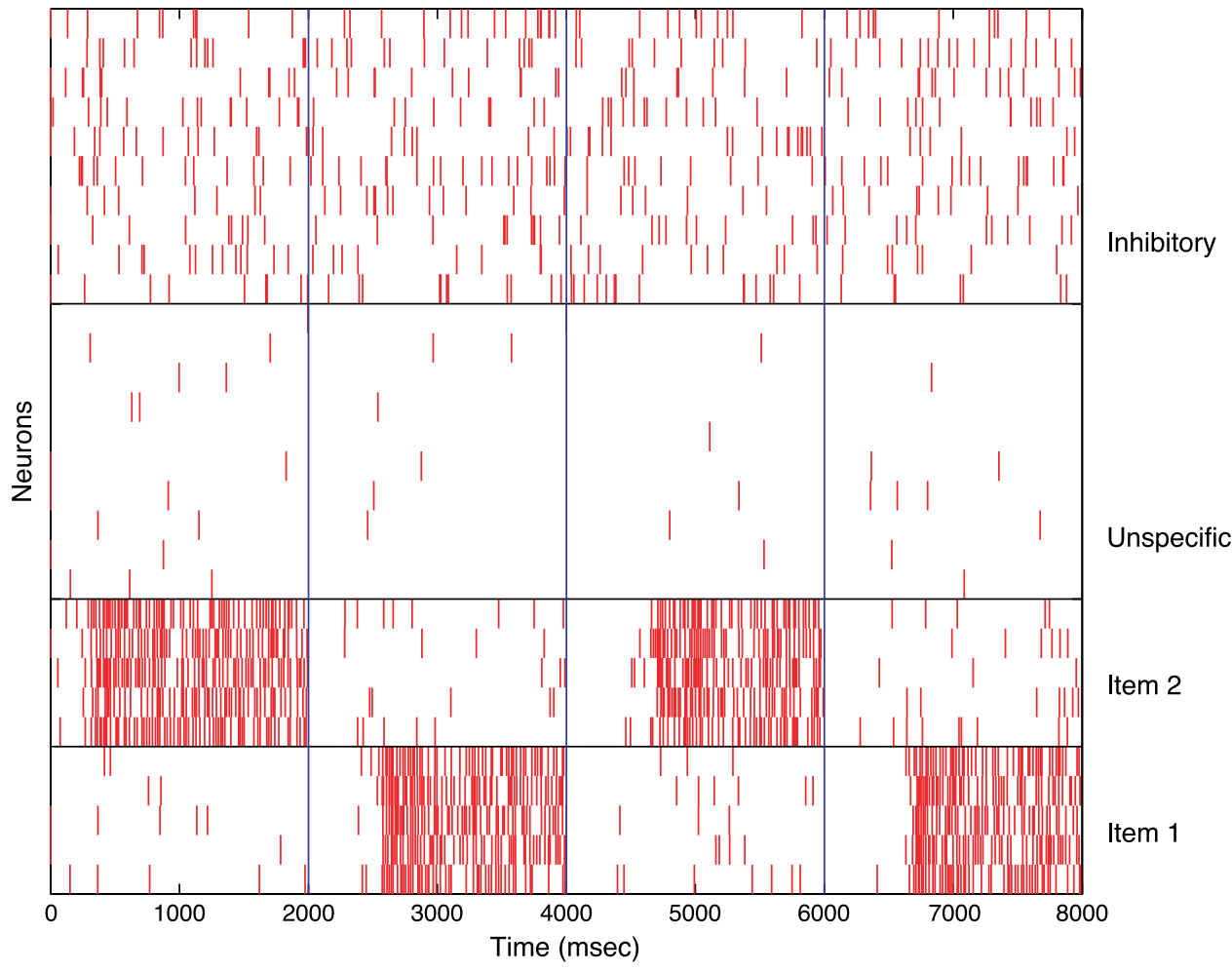


eters in parts of the brain that implement sequence memory may be different from those simulated.) In addition, other items stored in the same attractor might cause errors in retrieval unless there was a mechanism for preventing this. The error would arise if, for example, a fourth item was stored as an item by associative synaptic weight modification in a network, but was not part of a three-item sequence being remembered. After the first item in the sequence, with the second item still showing some adaptation, and the third item more adaptation, then the fourth item stored in the network would be retrieved, rather than the second item in the sequence. One way in which this is overcome is by the use of $B$ to facilitate just the items that are part of the current sequence, with the adaptation implementing the dynamics of the sequence order retrieval. This potential problem could also be overcome by making the associative synaptic modification that stores each item in the attractor network last for only a relatively short period. In particular, the problem would be solved if the long-term potentiation lasted for a shorter period than the period within which the network might be required to perform the learning and replay of another sequence. A simple scenario would be to have sufficient relatively short-lasting synaptic modification occurring during presentation of each item of the sequence during its initial presentation to set up the attractor for each item in the sequence and to make this synaptic modification last for just longer than the duration of the sequence to be remembered.

The type of sequence memory we describe is suitable for a short-term memory sequence mechanism. It would not incorporate and implement long-term frequently repeated fixed sequences of the type that might be involved in learning to play a piece of music and then playing the whole piece without the score. On the other hand, the sequence memory we describe would be suitable for a short-term scratchpad memory where only the most recent sequence would be remembered. In this respect, it would be similar to the recency part of human auditory-verbal short-term or working memory, in which different sequences of the same items can be correctly used, and only the most recent sequence is remembered (Baddeley, 1986). It is a remarkable property of the recency part of auditory-verbal short-term memory (and of the visuospatial scratchpad) that not just the items presented for memory, but the order in which the items were presented is a generic property of this type of memory (Baddeley, 1986). It is also a generic property of the type of sequence memory described here. It is also a property of auditory-verbal short-term or working memory that its capacity is strictly limited to $7 \pm 2$ items or "chunks" (Baddeley, 1986; Miller, 1956). Because of the way in which the adaptation must be stronger while the sequence is being replayed for later than earlier items in a sequence, and because the attractor that emerges after quiescence will be subject to noise effects, the maximum number of items in a sequence that could be remembered by the network described here is also likely to be limited to a few items. Indeed, we would be surprised if the model described here could remember many more than 7 items in a sequence. This limitation on the number of items in a sequence is in major contrast to the number of separate items that could be stored in the autoassociation network, which is in the order of the number of recurrent collateral synapses on each neuron if the representation is sparse (Rolls \& Deco, 2002; Rolls \& Treves, 1998). This would make the number of items in the network in the order of 5000-10,000, from which a subset of perhaps $7 \pm 2$ could be used in any sequence. Further, we note that the temporal period over which any sequence is recalled will be limited by the time constant of the adaptation process. Another property of the system described is that it would fail if an item in a sequence is repeated (e.g., 1-5-1-6, which would be recalled as 5-1-6). A solution to this potential problem is to use chunking (a strategy often used in human auditory-verbal short-term memory), by encoding the four-item sequence into, for example, 15-16. Another condition when recall of the sequence might not be correct would occur with this class of network if the external signal requesting the next item in the sequence occurs at one of the very short times when the adaptation variables for the different items happen to be equal. One such time occurs at approximately 2500 and 4800 in Figure 5 . Thus, this type of sequence memory might make occasional errors if the external signal comes at a particular time with respect to the adaptation processes, and this might be investigated experimentally in studies of human sequence memory.

We emphasize that the model makes a number of predictions. One that will allow it to be tested is whether in the attractor networks (perhaps in the prefrontal cortex) involved in short-term flexible sequence memory decrease their neuronal activity when the network progresses from one item in the sequence to the next. This is a property of the mechanism described, in that the transition between different attractors is produced by a decrease in neuronal activity in the attractors while

Figure 5. Synaptic adaptation mechanism. (A) Rate activity and average probability of transmitter release at the synapses in the different pools as a function of the time for a temporal sequence of two items. The two items were presented in the order item $2-$ item 1 at the start of the simulation period shown, and retrieval is demonstrated starting at $4000 \mathrm{msec}$. The two specific corresponding pools are shown. An external signal indicating that the network should move to the next item in the sequence is applied at time $=4000$ and time $=6000$ msec. (B) Rastergrams showing the spiking activity in the neurons in the different populations. 
the network is quenched. It is also an interesting and integral property of this type of short-term memory system with some adaptation that it automatically retrieves items in the same sequence in which they were presented, which is a striking characteristic of at least some human short-term memory systems, including the recency part of auditory-verbal short-term memory. It is thus a plausible suggestion that the recency effect part of auditory-verbal short-term memory (Baddeley, 1986) is implemented by a network with some of the mechanisms described here. It is also an integral property of the mechanism described here that it automatically starts its recall with the first item in the recently presented sequence of items, and this too is a characteristic property of the recency effect part of human auditoryverbal short-term memory. It is also a potentially telling similarity between the mechanism described here and some short-term memory systems in humans, including auditory-verbal short-term memory without explicit rehearsal and visuospatial short-term memory that both types fail if there is a long delay between presentation of the items to be remembered and the time when they are to be recalled. It is also of interest that the mechanism described here provides a mechanism by which rehearsal could be implemented, which is by repeating the external signal that causes quenching at regular intervals. In fact, this process is illustrated in Figures 2-5.

An application of a sequence network of the type described here apart from short-term sequence memory is central pattern generation (Grillner et al., 1998; Grillner, Wallen, Brodin, \& Lansner, 1991; Selverston, 1985), for which networks with dynamics of the type described here might be useful. Another application is in attentional and behavioral switching, and in reward reversal, wherein a signal of unexpected nonreward or punishment may switch an attractor network holding the current rule from one state to a different state that has not been recently active (Deco \& Rolls, in press).

We now contrast the implementation of a sequence memory described here with the more traditional method for storing a sequence of items in an attractor network, associating a time-delayed version of one item with the next item in the sequence (Kohonen, 1989; Sompolinsky \& Kanter, 1986; Hopfield, 1982; Kohonen, 1977). For that implementation, the recurrent collateral connections of the attractor network implement a fixed time delay, and the delayed representation of the first item is associated with the next item. Because of the time delay, this is an asymmetric network, with, in particular, the connections between neurons that represent different items in a sequence not symmetric as in a standard autoassociative network (Kohonen, 1989; Sompolinsky \& Kanter, 1986; Hopfield, 1982; Kohonen, 1977). A biological problem with this implementation of sequence memory is that the natural time delay in the recurrent collateral synapses is likely to be in the order of several milliseconds, so that when the sequence is recalled, the sequence will be recalled with a speed of several milliseconds between each item or several hundred items per second, which is rather fast. In comparison, the network described here is more graceful because it recalls successive items in a sequence, it can either be triggered, with no precise timing necessary, by an external signal that resets the network into quiescence (and the next item is then recalled) or the sequence can be automatically recalled at a speed that depends on how fast or slowly the adaptation builds up, which could be over in as long as many seconds. In the latter case, the items would be recalled in the correct sequence automatically without a trigger for each item and at a speed that depends on the underlying factors that determine how rapidly adaptation builds up. The short-term flexible sequence short-term memory we describe is also very different from the bird song sequence memory model of Drew and Abbott (2003), which learns a fixed sequence of items held in longterm memory, and an autoassociation network with asymmetric connections as above to implement a fixed sequence but noise to move attractor states from one item to the next (Buhmann \& Schulten, 1987).

\section{APPENDIX}

In this section, we give the mathematical equations that describe the spiking activity and synapse dynamics in the network, following in general the formulation described by Brunel and Wang (2001). Each neuron is described by an integrate-and-fire model. The subthreshold membrane potential $V(t)$ of each neuron evolves according to the following equation:

$$
C_{m} \frac{d V(t)}{d t}=-g_{m}\left(V(t)-V_{L}\right)-I_{\text {syn }}(t)
$$

where $I_{\text {syn }}(t)$ is the total synaptic current flow into the cell, $V_{\mathrm{L}}$ is the resting potential, $C_{\mathrm{m}}$ is the membrane capacitance, and $g_{\mathrm{m}}$ is the membrane conductance. When the membrane potential $V(t)$ reaches the threshold $\theta$, a spike is generated and the membrane potential is reset to $V_{\text {reset }}$. The neuron is unable to spike during the first $\tau_{\text {ref }}$ millisecond, which is the absolute refractory period. The total synaptic current is given by the sum of glutamatergic excitatory components (NMDA and AMPA) and inhibitory components (GABA). As described above, we consider that external excitatory contributions are produced through AMPA receptors $\left(I_{\mathrm{AMPA}, \text { ext }}\right)$, whereas the excitatory recurrent synapses operate through AMPA and NMDA receptors $\left(I_{\mathrm{AMPA}, \mathrm{rec}}\right.$ and $I_{\mathrm{NMDA}, \mathrm{rec}}$ ). The total synaptic current is therefore given by

$$
I_{\mathrm{syn}}(t)=I_{\mathrm{AMPA}, \mathrm{ext}}(t)+I_{\mathrm{AMPA}, \mathrm{rec}}(t)+I_{\mathrm{NMDA}, \mathrm{rec}}(t)+I_{\mathrm{GABA}}(t)
$$


where

$$
\begin{aligned}
I_{\mathrm{AMPA}, \mathrm{ext}}(t)= & g_{\mathrm{AMPA}, \mathrm{ext}}\left(V(t)-V_{E}\right) \sum_{j=1}^{N_{\mathrm{ext}}} s_{j}^{\mathrm{AMPA}, \mathrm{ext}}(t) \\
I_{\mathrm{AMPA}, \mathrm{rec}}(t)= & g_{\mathrm{AMPA}, \mathrm{rec}}\left(V(t)-V_{E}\right) \sum_{j=1}^{N_{E}} w_{j} s_{j}^{\mathrm{AMPA}, \mathrm{rec}}(t) \\
I_{\mathrm{NMDA}, \mathrm{rec}}(t)= & \frac{g_{\mathrm{NMDA}}\left(V(t)-V_{E}\right)}{\left(1+C_{M g^{++}} \exp (-0.062 V(t) / 3.57)\right)} \\
& \times \sum_{j=1}^{N_{E}} w_{j} s_{j}^{\mathrm{NMDA}}(t)
\end{aligned}
$$

In the preceding equations, $V_{\mathrm{E}}=0 \mathrm{mV}$ and $V_{1}=$ $-70 \mathrm{mV}$. The fractions of open channels, $s$, are given by:

$$
\begin{gathered}
\frac{d s_{j}^{\mathrm{AMPA}, \mathrm{ext}}(t)}{d t}=-\frac{s_{j}^{\mathrm{AMPA}, \mathrm{ext}}(t)}{\tau_{\mathrm{AMPA}}}+\sum_{k} \delta\left(t-t_{j}^{k}\right) \\
\frac{d s_{j}^{\mathrm{AMPA}, \mathrm{rec}}(t)}{d t}=-\frac{s_{j}^{\mathrm{AMPA}, \mathrm{rec}}(t)}{\tau_{\mathrm{AMPA}}}+\sum_{k} \delta\left(t-t_{j}^{k}\right) \\
\frac{d s_{j}^{\mathrm{NMDA}}(t)}{d t}=-\frac{s_{j}^{\mathrm{NMDA}}(t)}{\tau_{\mathrm{NMDA}, \mathrm{decay}}}+\alpha x_{j}(t)\left(1-s_{j}^{\mathrm{NMDA}}(t)\right) \\
\frac{d x_{j}(t)}{d t}=-\frac{x_{j}(t)}{\tau_{\mathrm{NMDA}, \mathrm{rise}}}+\sum_{k} \delta\left(t-t_{j}^{k}\right) \\
\frac{d s_{j}^{\mathrm{GABA}}(t)}{d t}=-\frac{s_{j}^{\mathrm{GABA}}(t)}{\tau_{\mathrm{GABA}}}+\sum_{k} \delta\left(t-t_{j}^{k}\right)
\end{gathered}
$$

where the sums over $k$ represent a sum over spikes emitted by presynaptic neuron $j$ at time $t_{j}^{k}$ The value of $\alpha=0.5 \mathrm{msec}^{-1}$.

The values of the conductances for pyramidal neurons were $g_{\mathrm{AMPA}, \mathrm{ext}}=2.08, g_{\mathrm{AMPA}, \mathrm{rec}}=0.104, g_{\mathrm{NMDA}}=0.328$, $g_{\mathrm{GABA}}=1.44 \mathrm{nS}$; for interneurons, $g_{\mathrm{AMPA}, \mathrm{ext}}=1.62$, $g_{\mathrm{AMPA}, \mathrm{rec}}=0.081, g_{\mathrm{NMDA}}=0.258$, and $g_{\mathrm{GABA}}=0.973 \mathrm{nS}$.

We used biologically realistic parameters (McCormick, Cannors, Lighthall, \& Prince, 1985). We take for both excitatory and inhibitory neurons a resting potential $V_{\mathrm{L}}=-70 \mathrm{mV}$, a firing threshold $\theta=-50 \mathrm{mV}$, and a reset potential $V_{\text {reset }}=-55 \mathrm{mV}$. The membrane capacitance, $C_{\mathrm{m}}$, is $0.5 \mathrm{nF}$ for the pyramidal neurons and $0.2 \mathrm{nF}$ for the inhibitory interneurons. The membrane leak conductance, $g_{\mathrm{m}}$, is $25 \mathrm{nS}$ for pyramidal cells and $20 \mathrm{nS}$ for interneurons. The refractory period, $\tau_{\text {ref }}$, is $2 \mathrm{msec}$ for pyramidal cells and $1 \mathrm{msec}$ for interneurons. Hence, the membrane time constant, $\tau_{\mathrm{m}},=C_{\mathrm{m}} / g_{\mathrm{m}}$ is $20 \mathrm{msec}$ for pyramidal cells and $10 \mathrm{msec}$ for interneurons.
We consider that the NMDA currents have a voltage dependence that is controlled by the extracellular magnesium concentration (Jahr \& Stevens, 1990), $C_{\mathrm{Mg}^{++}}=$ $1 \mathrm{mM}$. We neglect the rise time of both AMPA and GABA synaptic currents because they are typically very short $(<1 \mathrm{msec})$. The rise time for NMDA synapses is $\tau_{\mathrm{NMDA}, \text { rise }}=2$ msec (Spruston, Jonas, \& Sakmann, 1995; Hestrin, Sah, \& Nicoll, 1990). All synapses have a time delay of $0.5 \mathrm{msec}$. The time constant for AMPA synapses is $\tau_{\mathrm{AMPA}}=2 \mathrm{msec}$ (Spruston et al., 1995; Hestrin et al., 1990 ), for NMDA synapses, $\tau_{\mathrm{NMDA} \text {, decay }}=100 \mathrm{msec}$ (Spruston et al., 1995; Hestrin et al., 1990), and for GABA synapses, $\tau_{\mathrm{GABA}}=10 \mathrm{msec}$ (Xiang, Huguenard, \& Prince, 1998; Salin \& Prince, 1996). The synaptic conductivities for each receptor type were taken from Brunel and Wang (2001) and were adjusted using a mean field analysis to be approximately $1 \mathrm{nS}$ in magnitude and were consistent with experimentally observed values (Destexhe, Meinen, \& Sejnowski, 1998).

\section{Acknowledgments}

This work was supported by the German Ministry of Research, BMBF grant 01IBC01A, and by the UK Medical Research Council. Gustavo Deco was supported by Institucion Catalana de Recerca i Estudis Avanats (ICREA).

Reprint request should be sent to Edmund T. Rolls, Department of Experimental Psychology, University of Oxford, South Parks Road, Oxford OX1 3UD, UK, or via e-mail: edmund. rolls@psy.ox.ac.uk, URL: www.cns.ox.ac.uk.

\section{REFERENCES}

Abeles, A. (1991). Corticonics. New York: Cambridge University Press.

Amit, D., \& Brunel, N. (1997). Model of global spontaneous activity and local structured activity during delay periods in the cerebral cortex. Cerebral Cortex, 7, 237-252.

Averbeck, B., Chafee, M., Crowe, D., \& Georgopoulos, A. (2002). Parallel processing of serial movements in prefrontal cortex. Proceedings of the National Academy of Sciences. U.S.A. 99. 13172-13177.

Baddeley, A. (1986). Working memory. New York: Oxford University Press.

Battaglia, F., \& Treves, A. (1998). Stable and rapid recurrent processing in realistic autoassociative memories. Neural Computation, 10, 431-450.

Brunel, N., \& Wang, X. (2001). Effects of neuromodulation in a cortical networks model of object working memory dominated by recurrent inhibition. Lournal of Computational Neuroscience, 11, 63-85.

Buhmann, J., \& Schulten, K. (1987). Noise-driven temporal association in neural networks. Europhysics Letters, 4, 1205-1209.

Compte, A., Constantinidis, C., Tegner, J., Raghavachari, S., Chafee, M. V., Goldman-Rakic, P. S., \& Wang, X. J. (2003). Temporally irregular mnemonic persistent activity in prefrontal neurons of monkeys during a delayed response task. Journal of Neurophysiology, 90, 3441-3454.

Conway, C., \& Christiansen, M. (2001). Sequential learning in non-human primates. Trends in Cognitive Sciences, 5 , 539-546. 
Corchs, S., \& Deco, G. (2002). Large-scale neural model for visual attention: Integration of experimental single cell and fMRI data. Cerebral Cortex, 12, 339-348.

Dayan, P., \& Abbott, L. (2002). Theoretical neuroscience: Computational and mathematical modeling of neural systems. Boston: MIT Press.

Deco, G., \& Lee, T. (2002). A unified model of spatial and object attention based on inter-cortical biased competition. Neurocomputing, 44-46, 775-781.

Deco, G., \& Rolls, E. T. (2003). Attention and working memory: A dynamical model of neuronal activity in the prefrontal cortex. European Journal of Neuroscience, 18, 2374-2390.

Deco, G., \& Rolls, E. T. (in press). Synaptic and spiking dynamics underlying reward reversal in the orbitofrontal cortex. Cerebral Cortex.

Deco, G., \& Zihl, J. (2001). Top-down selective visual attention: A neurodynamical approach. Visual Cognition, 8, 119-140.

Dell, G., Burger, L., \& Svec, W. (1997). Language production and serial order: A functional analysis and a model. Psychological Review, 104, 123-147.

Destexhe, A., Mainen, Z., \& Sejnowski, T. (1998). Kinetic models of synaptic transmission. In C. Koch \& I. Segev (Eds.), Methods in neural modeling: From ions to networks (2nd ed., pp. 1-25). Cambridge: MIT Press.

Drew, P. J., \& Abbott, L. F. (2003). A neural network model of sensoritopic maps with predictive short-term memory properties. Journal of Neurophysiology, 89, 2697-2706.

Fuster, J. (2000). Executive frontal functions. Experimental Brain Research, 133, 66-70.

Giugliano, M., La Camera, G., Rauch, A., Luescher, H.-R., \& Fusi, S. (2002). Non-monotonic current-to-rate response function in a novel integrate-and-fire model neuron. In J. Dorronsoro (Ed.), Proceedings of ICANN 2002, LNCS 2415 , (pp. 141-146). New York: Springer-Verlag.

Goldman-Rakic, P. (1987). Circuitry of primate prefrontal cortex and regulation of behavior by representational memory. In F. Plum \& V. Mountcastle (Eds.), Handbook of physiology-The nervous system (pp. 373-417). Bethesda, MD: American Physiological Society.

Grillner, S., Ekeberg, El-Manira, A., Lansner, A., Parker, D., Tegner, J., \& Wallen, P. (1998). Intrinsic function of a neuronal network-A vertebrate central pattern generator. Brain Research Reviews, 26, 184-197.

Grillner, S., Wallen, P., Brodin, L., \& Lansner, A. (1991). Neuronal network generating locomotor behavior in lamprey: Circuitry, transmitters, membrane properties, and simulation. Annual Review of Neuroscience, 14, 169-199.

Hansel, D., Mato, G., Meunier, C., \& Neltner, L. (1998). On numerical simulations of integrate-and-fire neural networks. Neural Computation, 10, 467-483.

Hestrin, S., Sah, P., \& Nicoll, R. (1990). Mechanisms generating the time course of dual component excitatory synaptic currents recorded in hippocampal slices. Neuron, 5, 247-253.

Hopfield, J. J. (1982). Neural networks and physical systems with emergent collective computational abilities.
Proceedings of the National Academy of Sciences, U.S.A., 79, 2554-2558.

Jahr, C., \& Stevens, C. (1990). Voltage dependence of NMDA-activated macroscopic condunctances predicted by single-channel kinetics. Journal of Neuroscience. 10. 3178-3182.

Kohonen, T. (1977). Associative memory: A system theoretical approach. New York: Springer-Verlag.

Kohonen, T. (1989). Self-organization and associative memory (3rd ed.). Berlin: Springer-Verlag.

Lashley, K. (1951). Cerebral mechanisms in behavior. New York: Wiley.

Liu, Y., \& Wang, X.-J. (2001). Spike-frequency adaptation of a generalized leaky integrate-and-fire model neuron. Journal of Computational Neuroscience, 10, 25-45.

Luria, A. (1966). Higher cortical functions in man. New York: Basic Books.

McCormick, D., Connors, B., Lighthall, J., \& Prince, D. (1985). Comparative electrophysiology of pyramidal and sparsely spiny stellate neurons in the neocortex. Journal of Neurophysiology, 54, 782-806.

Miller, G. A. (1956). The magic number seven, plus or minus two: Some limits on our capacity for the processing of information. Psychological Review, 63, $\underline{81-93 .}$

Petrides, M. (1991). Functional specialization within the dorsolateral frontal cortex for serial order memory. Proceedings of the Royal Society London Series B, 246, 299-306.

Rauch, A., La Camera, G., Luescher, H.-R., Senn, W., \& Fusi, S. (2003). Neocortical pyramidal cells respond as integrateand-fire neurons to in vivo-like input currents. Journal of Neurophysiology . 90, 1598-1612.

Rolls, E. T., \& Deco, G. (2002). Computational neuroscience of vision. Oxford: Oxford University Press.

Rolls, E. T., \& Treves, A. (1998). Neural networks and brain function. Oxford: Oxford University Press.

Salin, P., \& Prince, D. (1996). Spontaneous GABA-A receptor mediated inhibitory currents in adult rat somatosensory cortex. Lournal of Neurophysiologv, 75, 1573-1588.

Selverston, A. I. (Ed.). (1985). Model neural networks and behavior. New York: Plenum.

Sompolinsky, H., \& Kanter, I. (1986). Temporal association in asymmetric neural networks. Physical Review Letters, 57. 2861-2864.

Spruston, N., Jonas, P., \& Sakmann, B. (1995). Dendritic glutamate receptor channel in rat hippocampal CA3 and CA1 pyramidal neurons. Journal of Physiology, 482, 325-352.

Wilson, F. A. W., O'Scalaidhe, S. P., \& Goldman-Rakic, P. (1994). Functional synergism between putative gamma-aminobutyrate-containing neurons and pyramidal neurons in prefrontal cortex. Proceedings of the National Academy of Sciences, U.S.A., 91, 4009-4013.

Xiang, Z., Huguenard, J., \& Prince, D. (1998). GABA-A receptor mediated currents in interneurons and pyramidal cells of rat visual cortex. Journal of Physiology, 506, 715-730. 